\title{
La mort et le don d'organes
}

\author{
Olivier Soubrane
}

L

e don d'organes est l'objet d'un débat social, intellectuel et juridique. Le nombre de dons permettant de réaliser des prélèvements d'organes chez des personnes décédées est en diminution depuis quelques années. La principale conséquence en est la baisse du nombre de transplantations d'organes comme le cœur, le foie, les poumons ou les reins. Une des causes à l'origine de cette diminution du prélèvement d'organes chez des personnes décédées, est représentée par les oppositions familiales à cette procédure. Différents groupes sociaux s'inquiètent, à juste titre, de l'augmentation de ces refus: en premier lieu, les médecins et les chirurgiens qui soignent les malades en attente d'une greffe d'organe et pour lesquels la transplantation représente le seul espoir de guérison ou de survie. Quelles sont donc les raisons à l'origine des oppositions au don d'organes? Pour tenter de les cerner, il est avant tout nécessaire d'évoquer le contexte dans lequel intervient ce don: la mort.

La mort éteint la conscience qui a tenté de la représenter, de la conceptualiser: la mort est toujours et d'abord celle de l'autre car je ne peux expérimenter ma propre mort, même si ma mort ne peut être que la mienne [1]. Irreprésentable, inexpérimentable, la mort est pourtant la condition de la vie, car ne peut vivre que ce qui est mortel: "en tant qu'Arche du Rien, la mort est l'abri de l'être» [2]. Concernant le don d'organes, les causes de la mort qui peuvent engager un tel processus de

vent d'un événement brutal : traumatisme crânien, hémorragie cérébrale survenant chez une personne jusquelà en bonne santé. Cette soudaineté fait que le mort et ses proches n'ont pas eu le temps de cette mort et de la comprendre: "on n'a pas eu le temps de mourir, on est déjà mort » disait Husserl [3]. Nous n'avons pas le temps de vaincre cette mort - au sens métaphysique - que l'on nous demande de surmonter la finitude individuelle par l'acte de donation. Les difficultés sont donc grandes. En effet, différents obstacles potentiels au principe du don d'organes peuvent, dans ce contexte, être distingués. Ils concernent surtout la famille du défunt qui seule «vit" la mort, la perte. Il peut exister un doute ou un déni de la réalité de la mort. Des critères scientifiques établissent cette mort avec certitude: sont-ils toujours perçus ou perceptibles par tous avec cette certitude? La crainte d'une atteinte de l'intégrité corporelle, réelle ou fantasmatique, risque de perturber le rite des funérailles, de l'inhumation, et ainsi de compromettre le deuil. Si le corps réel du défunt, celui de la parole, de la jouissance - et finalement celui de la pulsion de mort - n'est plus, il est essentiel de respecter, vis-à-vis de la famille, le corps symbolique et imaginaire. Enfin, un obstacle paradoxal au don d'organes est la publicité ou la promotion que certains sont tentés de lui faire. En effet, essayer d'imposer le don d'organes, y inciter, forcer son message par des campagnes médiatiques, en faire l'apologie tapageuse en stigmatisant le refus de donner - ce dernier étant parfois injuste- ment assimilé à une "éthique de l'égoïsme» [4] - témoignent d'une incompréhension du principe du don et peuvent conduire à son rejet. Tout discours sur le don d'organes devrait se fonder sur la compréhension de la nature profonde du don lui-même et sa conception morale.

Ainsi, nous voyons bien aujourd'hui que confusions, malentendus, et surtout manque de réflexion du public et des médecins vis-à-vis de la mort et du concept du don risquent de compromettre l'existence naturellement fragile du don d'organes, malmené entre les considérations juridiques techniques et les mirages de certaines philosophies utilitaristes. Au-delà des choix que la société a faits et qu'elle est susceptible, ou non, de modifier, certaines considérations morales sur le don d'organes méritent aujourd'hui notre attention. Les deux aspects fondamentaux de cette réflexion concernent la mort, nous l'avons vu, et cette connexion inter-humaine particulière qu'est le don. «Donner et recevoir sont des conduites premières qui appartiennent à un langage corporel antérieur à la parole» disait Jean Starobinski [5]. Le don d'un organe, son prélèvement chez un être humain décédé et sa transplantation chez un autre être humain receveur n'est qu'un aspect infime - mais combien singulier - du don, de ces dons, de ces échanges d'un individu à l'autre qui déterminent toute notre vie, en font sa structure et sa mémoire. Accepter le don d'organes, c'est aussi, déjà, accepter la mort, ou plutôt s'orienter vers l'acceptation future de l'encore inacceptable. Ce dernier point souligne l'extrême importance du discours 
public sur le don d'organes qui doit être réflexion plutôt qu'exhortation. En effet, parler de notre mort, de celle de nos proches, c'est aller au plus profond de nous-mêmes et toute légèreté du discours sur la mort ou toute tentative de culpabilisation vis-à-vis du don sont dérisoires et inadaptées. La réflexion est nécessaire à tous et notamment aux médecins eux-mêmes, à ceux qui sont au contact des familles et qui ont à leur parler et à les écouter. Leur réflexion mais aussi leur formation dans ce sens doit être à la hauteur de la gravité de ce qu'ils côtoient: la mort des uns et la douleur des autres. Le don n'est pas indispensable mais il est dispensateur. Il n'est pas juge ou témoin d'un groupe ou d'une société: il fait partie de la dynamique de ces groupes. C'est dans la liberté de donner ou de ne pas le faire que l'on mesure la valeur et la symbolique du don. La liberté de l'homme est dans sa liberté de choix, et l'acceptation et le refus du don existent et se définissent l'un par l'autre, le second établissant la dimension du premier. Enfin, l'ultime caractéristique du don d'organes lui confère aussi une dimension admirable: le receveur du don c'est l'autre, l'inconnu avec lequel n'existe aucun lien affectif ni libidinal. On pourrait s'interroger sur la nature réelle, le déterminisme du don d'organes, ses ressorts: s'agit-il de miséricorde, de cette capacité qu'auraient un homme, une famille, frappés par la douleur de la perte, d'être sensibles au malheur d'autrui? S'agirait-il de quelque chose de plus mystérieux encore? La signification profonde de la donation est sans nul doute très complexe.

Le don d'organes est précieux, allant au-delà de l'activité de transplantation d'organes et des progrès scientifiques et techniques. En effet, le don de soi - qu'il s'agisse en l'occurrence du don d'organes - s'oppose au néant de la mort des philosophes et donner sans contrepartie aucune, sans échange ni réciprocité - don suprême - est l'aboutissement de la vie humaine dans une perspective ontogénique: celle où le donateur et le donataire maintiennent la continuité de l'être et de son prochain. Par cette filiation organique, le donataire, receveur du don, devient doué par la grâce de l'autre, véritable sublimation de la non-mort, du nonanéantissement, à laquelle la famille et les proches du donateur sont indéfectiblement et définitivement liés. En cela, chacun devient responsable de l'autre, de tous les autres

\section{RÉFÉRENCES}

1. Derrida J. Donner la mort. In: L'éthique du don. Paris: Métailié-Transition, 1992 : 45.

2. Heidegger M. La chose. Paris : Gallimard, 1958: 213 .

3. Husserl E. Le monde anthropologique. In: Naître et mourir. Paris: Alter, 1993: 27190

4. Got C. Contre une éthique de l'égoïsme. Le Monde, $1^{\text {er }}$ juin 1994.

5. Starobinski J, Largesse. Réunion des Musées Nationaux, Paris, 1994: 8.

\section{O. Soubrane}

Professeur des universités, chirurgien des hôpitaux, Service de chirurgie générale et digestive, hopital Cochin, 27, rue du Faubourg-Saint-Jacques, 75014, Paris, France.

\section{TIRÉS À PART}

O. Soubrane.

\section{$22^{\mathrm{e}}$ Symposium Européen Hormones et Régulation cellulaire avec l'aide de l'INSERM Mont-Sainte-Odile (Alsace, France) 3-6 octobre 1997}

L'AMP cyclique de la membrane plasmique au noyau : adénylyl cyclases, protéines $\mathbf{G}$ trimériques, phosphodiestérases, régulation des gènes, régulations complexes et pathologiques.

J.A. Beavo (US) ; L. Birnbaumer (USA) ; J. Bockaert (F) ; A. Danchin (F) ; J. Dumont (B) ; R. Fischmeister (F) ; P. Gierschik (G) ; A. Gilchrist (US) ; T. Guderman (G) ; M. Houslay (UK) ; M. Lohse (G) ; S. Lotersztajn (F) ; C. Lugnier (F) ; N. Mons (F) ; M. Montminy (US) ; A. Munnich (F) ; S. Offermanns (G) ; E. Pays (B) ; J. Saez (F) ; D. Storm (US) ; P. Sassone Corsi (F) ; G. Schütz (G) ; E. Tzavara (Gr).

\section{Date limite de soumission des résumés et d'inscription : 15 juin 1997}

Information et inscription :

Dr J. Hanoune, Symposium Ste Odile, INSERM U. 99, Hôpital Henri-Mondor 94010 CRÉTEIL, France. Fax : 33148980908 @mail : hanoune@im3.inserm.fr. 